\title{
Correction to: Thirty years of small business economics: a bibliometric overview
}

\author{
H. Kent Baker • Satish Kumar • Nitesh Pandey
}

Published online: 18 June 2020

(C) Springer Science+Business Media, LLC, part of Springer Nature 2020

\section{Correction to: Small Bus Econ}

$$
\text { https://doi.org/10.1007/s11187-020-00342-y }
$$

The original version of this article unfortunately contained a mistake in some of the data in the Table 3. The corrected Table 3 is shown below.

Publisher's note Springer Nature remains neutral with regard to jurisdictional claims in published maps and institutional affiliations.
The online version of the original article can be found at https://doi.org/10.1007/s11187-020-00342-y

H. K. Baker $(\bowtie)$

Department of Finance and Real Estate, Kogod School of Business, American University, 4400 Massachusetts Avenue, NW, Washington, DC 20016, USA

e-mail:kbaker@american.edu

S. Kumar · N. Pandey

Department of Management Studies, Malaviya National Institute of Technology, Jaipur, Rajasthan 302017, India 
Table 3 Top 20 SBE authors and their affiliated institutions between 1989 and 2018

No. of publications cited

\begin{tabular}{|c|c|c|c|c|c|c|c|c|c|c|}
\hline Author & Current Affiliation & TA & TCA & $\mathrm{TC}$ & $\mathrm{C} / \mathrm{A}$ & $\mathrm{C} / \mathrm{CA}$ & $h$ & $\geq 500$ & $\geq 100$ & $\geq 50$ \\
\hline Ács, Z. J. & George Mason University & 41 & 39 & 3122 & 76.15 & 80.05 & 23 & 1 & 10 & 17 \\
\hline Audretsch, D. B. & Indiana University & 31 & 30 & 2264 & 73.03 & 75.47 & 22 & 1 & 7 & 16 \\
\hline Thurik, R. & Erasmus School of Economics & 26 & 26 & 4020 & 154.62 & 154.62 & 16 & 1 & 9 & 15 \\
\hline Wright, M. T. & Imperial College London & 19 & 19 & 1165 & 61.32 & 61.32 & 16 & 0 & 3 & 6 \\
\hline Link, A. N. & The University of North Carolina at Greensboro & 18 & 18 & 810 & 45.00 & 45.00 & 12 & 0 & 2 & 4 \\
\hline Carlsson, B. & Case Western Reserve University & 14 & 14 & 1093 & 78.07 & 78.07 & 10 & 1 & 2 & 5 \\
\hline Storey, D. J. & University of Sussex & 13 & 13 & 1069 & 82.23 & 82.23 & 9 & 0 & 4 & 7 \\
\hline Henrekson, M. & Research Institute of Industrial Economics & 13 & 13 & 884 & 68.00 & 68.00 & 10 & 0 & 3 & 5 \\
\hline Cowling, M. & University of Derby & 13 & 13 & 470 & 36.15 & 36.15 & 10 & 0 & 2 & 3 \\
\hline Carree, M. A. & Maastricht University & 12 & 12 & 1214 & 101.17 & 101.17 & 10 & 1 & 3 & 4 \\
\hline Reynolds, P. D. & Aston University & 11 & 11 & 1892 & 172.00 & 172.00 & 10 & 1 & 5 & 6 \\
\hline van Stel, A. J. & Trinity College Dublin & 11 & 11 & 1017 & 92.45 & 92.45 & 10 & 0 & 4 & 5 \\
\hline Fritsch, M. & Friedrich Schiller Universität Jena & 11 & 11 & 513 & 46.64 & 46.64 & 8 & 0 & 2 & 4 \\
\hline Parker, S. C. & Western University & 10 & 10 & 368 & 36.80 & 36.80 & 8 & 0 & 1 & 2 \\
\hline Terjesen, $\mathrm{S}$. & American University & 10 & 10 & 368 & 36.80 & 36.80 & 7 & 0 & 1 & 3 \\
\hline Bosma, N. S. & Utrecht University & 9 & 9 & 1371 & 152.33 & 152.33 & 8 & 1 & 3 & 5 \\
\hline Braunerhjelm, P. & The Royal Institute of Technology (KTH) & 9 & 9 & 944 & 104.89 & 104.89 & 6 & 1 & 2 & 4 \\
\hline Wagner, J. & Leuphana Universität Lüneburg & 9 & 9 & 548 & 60.89 & 60.89 & 8 & 0 & 3 & 3 \\
\hline Vivarelli, M. & Università Cattolica del Sacro Cuore & 9 & 8 & 509 & 56.56 & 63.63 & 7 & 0 & 1 & 6 \\
\hline Colombo, M. G. & Politecnico di Milano & 9 & 9 & 397 & 44.11 & 44.11 & 9 & 0 & 0 & 1 \\
\hline
\end{tabular}

Note: This table lists the top 20 SBE authors and their affiliated institutions where $\mathrm{TA}=$ total articles, $\mathrm{TC}=$ total citations, $\mathrm{TCA}=$ total cited articles, TC/TA $=$ cites per article, TC/TCA $=$ cited per cited article, $h=h$-index, and $\geq 500, \geq 100$, and $\geq 50=$ the number of articles receiving at least 500,100, and 50 citations, respectively. The table excludes documents categorized by Scopus as letters, erratum, and undefined 